\title{
Synthesis of UV-curable silane-coupling agent as an adhesion promoter
}

\author{
Kuo-Huai Kuo a, Wen-Yen Chiu ${ }^{\text {a,b,c,*, Kuo-Huang Hsieh }}{ }^{\text {a,b }}$ \\ a Department of Chemical Engineering, National Taiwan University, Taipei 106, Taiwan \\ ${ }^{\mathrm{b}}$ Institute of Polymer Science and Engineering, National Taiwan University, Taipei 106, Taiwan \\ ${ }^{c}$ Department of Materials Science and Engineering, National Taiwan University, Taipei 106, Taiwan
}

\section{A R T I C L E I N F O}

\section{Article history:}

Received 7 June 2008

Received in revised form 13 August 2008

Accepted 19 August 2008

\section{Keywords:}

UV-curable silane

Adhesion

Black matrix

\begin{abstract}
A B S T R A C T
In color filter industry, a problem was found that the adhesion strength between glass substrate and black matrix was largely decreased after ITO sputtering process. In order to solve this problem, a new UV-curable silane-coupling agent (UV-SCA) was synthesized to be an adhesion promoter, which was synthesized by the reaction between the hydroxyl group of bisphenol A epoxy diacrylate and the isocyanate group of 3(isocyanatopropyl)-triethoxysilane. The adhesion strength between glass substrate and black film, which was prepared from the carbon black photo-resist with or without the addition of UV-SCA, was determined by a tensile/compression strength tester, and the effect of UV-SCA on the adhesion strength before and after ITO sputtering process was also discussed.
\end{abstract}

(C) 2008 Elsevier B.V. All rights reserved.

\section{Introduction}

Organo-functional silanes have often been used as coupling agents in composite materials. They were widely used to treat inorganic substrates, such as metal, glass, and minerals, in order to enhance the compatibility between an inorganic substrate and a polymeric matrix [1-7].

Silane-coupling agents containing acrylate and methacrylate groups were useful in several different ways. For example, 3-acryloxypropyltrimethoxysilane (APMS) and 3-methacryloxypropyltrimethoxysilane (MPS), containing acrylate and methacrylate functional groups on the alkyl chain, have been used in polymer/glass fiber composites because they can enhance both the mechanical properties and the optical transference of composites $[8,9]$. In addition, many studies have reported the preparation of organic-inorganic composites by in situ polymerization of metal alkoxides in organic polymers, derived from a methacrylic or an acrylic group [10-14]. For example, Saitoh et al. [14] prepared a thermo-responsive glass capillary by coupling APMS on the inner surface of glass capillary and a subsequent radical polymerization of $\mathrm{N}$-isopropylacrylamide and methylenebis(acrylamide). In some specific fields, APMS and MPS were used for ultraviolet (UV) coatings applications $[2,15,16]$. Hagberg et al. [16] produced a nanopattern on silicon wafer substrate by using imprint lithography method. They used APMS to prepare an adhesion promoter layer in

\footnotetext{
* Corresponding author at: Department of Chemical Engineering, National Taiwan University, Taipei 106, Taiwan. Tel.: +886 23623259; fax: +886223623040.

E-mail address: ycchiu@ntu.edu.tw (W.-Y. Chiu).
}

order to improve the adhesion strength between the silicon wafer and photo-resist. Chiang and Hsieh [2] investigated the effect of different types of organo-functional silanes in the cationic polymerization of epoxide resin on the epoxide adhesion strength to indium tin oxide (ITO) substrate. They found that the adhesion strength was improved by using vinyltrimethoxysilane, APMS, and MPS as adhesion promoters.

In color filter industry, a problem was found that the adhesion strength between the glass and black matrix was largely decreased after ITO sputtering process. To overcome this problem, in this study, a new silane-coupling agent was synthesized to be an adhesion promoter. This silane-coupling agent was synthesized by the reaction between bisphenol A epoxy diacrylate (EPA) and 3-(isocyanatopropyl)-triethoxysilane (ICPTES); it also exhibited UV-curable property. The schematic diagram of the synthesis of UVcurable silane-coupling agent (UV-SCA) was shown in Scheme 1. In addition, the UV-SCA is non-toxic to the human body because of its large molecular weight and non-volatile property, and it can replace some volatile silane-coupling agents, such as APMS and MPS, in the photo-resist application. In this work, two carbon black photo-resists with or without the addition of UV-SCA were taken to prepare black films on the glass substrate. The black films were cured upon UV-irradiation, and subsequently post-cured at $200{ }^{\circ} \mathrm{C}$. The schematic illustrations of photo-polymerization upon UV-irradiation and coupling reaction between alkoxy group of UVSCA and glass substrate during post-cured process were shown in Scheme 2, respectively. The adhesion strength between the glass substrate and black film was determined by a tensile/compression strength tester, and the effect of UV-SCA on the adhesion strength before and after ITO sputtering process was also discussed. 


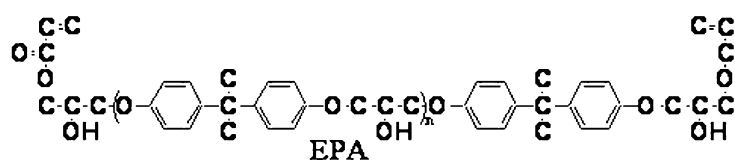

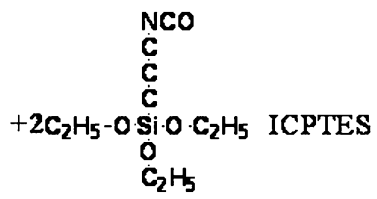

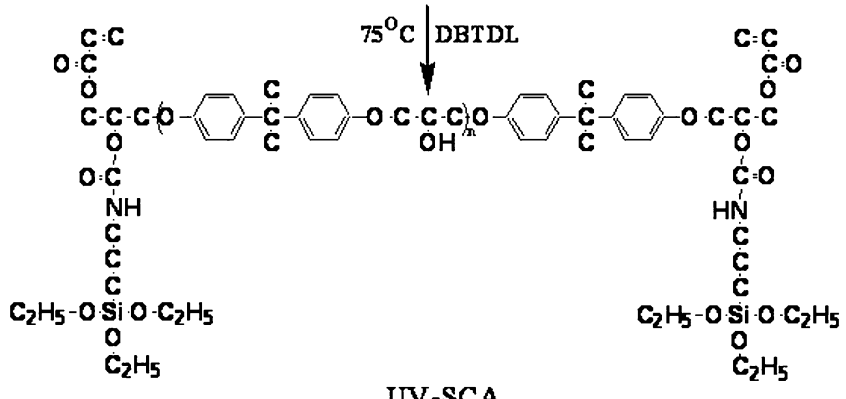

UV-SCA

Scheme 1. Synthesis of UV-curable silane-coupling agent (UV-SCA).

\section{Experimental}

\subsection{Materials}

Bisphenol-A epoxy diacrylate (AGI Corporation), 3-(isocyanatopropyl)triethoxysilane (ICPTES, Aldrich), di- $n$-butyltin dilaurate (DBTDL, TCI), propylene glycol monomethyl ether acetate (PGMEA, Grand Chemical), and two carbon black photo-resists (CB PRs): BK5017 and ADK L4, were all used as received. The CB PRs comprised carbon black, alkali-soluble resins, multi-functional monomers or oligomers, photo-initiator, and solvent. The solid content in CB PR was approximately $20 \mathrm{wt} \%$.

\subsection{Synthesis of UV-curable silane-coupling agen}

EPA and ICPTES were dissolved in PGMEA, in which the solid content was ca. $40 \mathrm{wt} \%$. The molar ratio of EPA to ICPTES was 1:2, and DBTDL was used as a catalyst. The reaction mixture was stirred by a magnetic stirring bar and kept at $75^{\circ} \mathrm{C}$ in a nitrogen atmosphere until the isocyanate group monitored by a FTIR was consumed completely to obtain the reaction product.

\subsection{Structural analysis}

Functional groups of UV-SCA were characterized by a FTIR (DIGILAB FTS4000) in the range of $4000-900 \mathrm{~cm}^{-1}$. A 500-MHz ${ }^{1} \mathrm{H}$ NMR spectrometer (Bruker Avance-

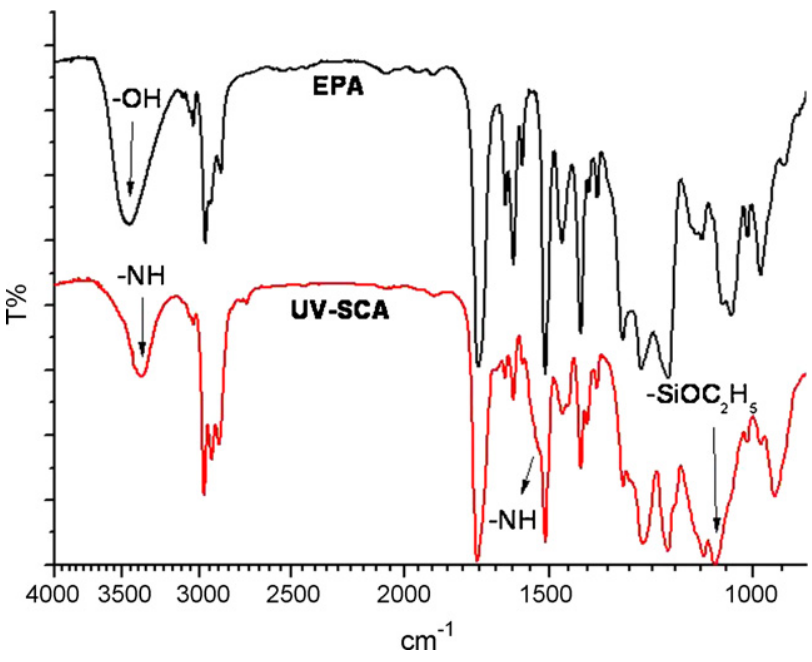

Fig. 1. FTIR spectra of EPA and UV-SCA
$500 \mathrm{MHz}$ FT NMR) was used to obtain NMR spectra by using $\mathrm{CDCl}_{3}$ as a solvent, and was used to identify the composition of UV-SCA.

\subsection{Adhesion strength}

A CB PR with or without $1 \mathrm{wt} \%$ UV-SCA (based on the total solid of CB PR) was dropped onto a glass substrate to prepare a black film by a spin coating process with $900 \mathrm{rpm}$ for $10 \mathrm{~s}$. This black film was pre-cured at $50^{\circ} \mathrm{C}$ for $2 \mathrm{~min}$, exposed to UV light of $200 \mathrm{~mJ} \mathrm{~cm}^{-2}$, and subsequently post-cured at $200^{\circ} \mathrm{C}$ for $30 \mathrm{~min}$ Finally, the ITO film was coated onto the above black film by a sputtering process.

The adhesion strength between the glass substrate and black film was determined by a tensile/compression strength tester (QC-503B1 universal material testing machine, Cometech) and the experimental procedure was described as follows: An aluminum pin was bound onto the above black film with or without the ITO sputtering process by applying the adhesive epoxy resin. It was then cured at $200^{\circ} \mathrm{C}$ for five minutes to prepare a pull test specimen. The adhesion strength of the specimen was measured at a pull rate of $3 \mathrm{~mm} \mathrm{~min}^{-1}$ until the aluminum pin was peeled off. The pull test specimen and the test method to determine the adhesion

(a) before UV irradiation
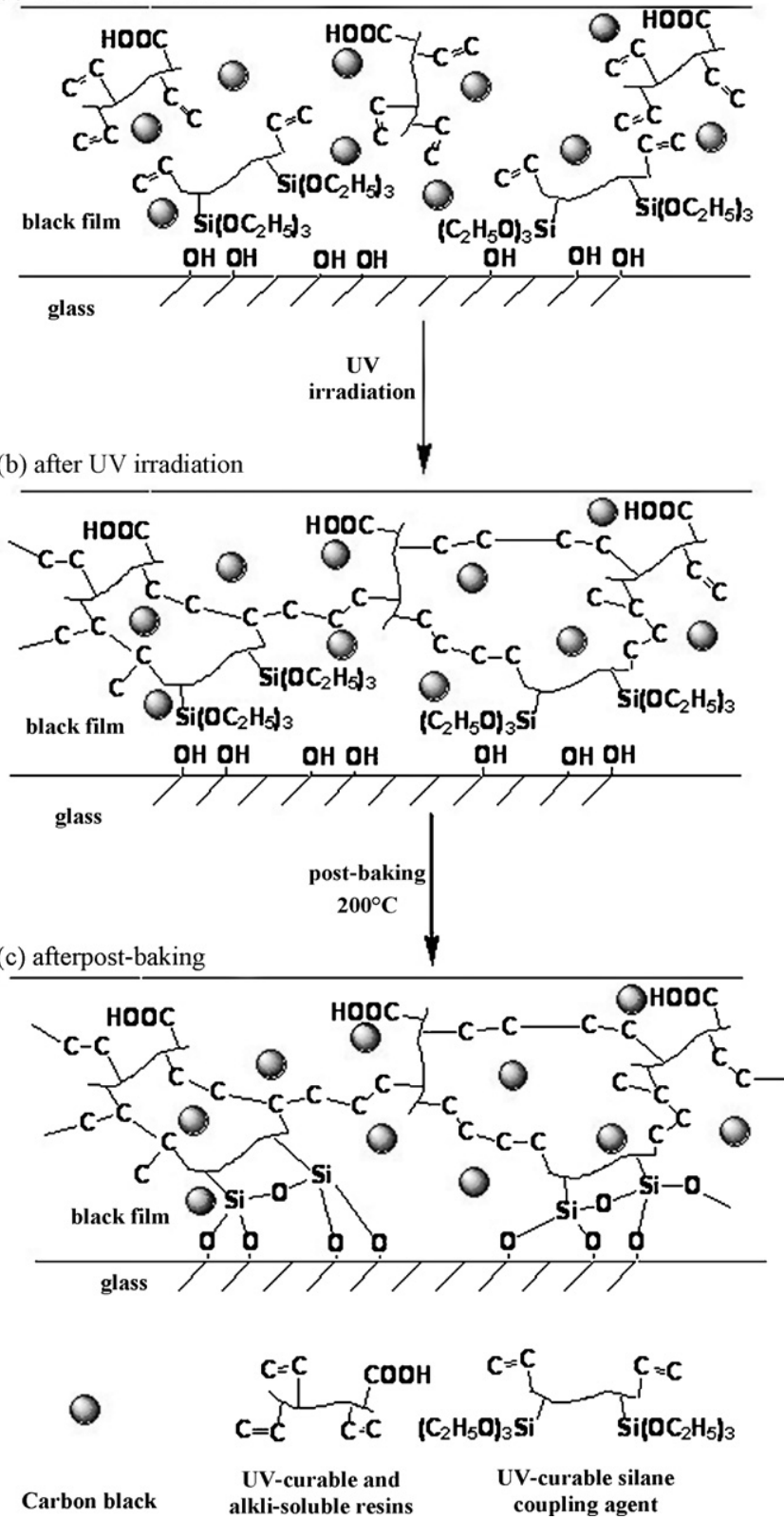

Scheme 2. The black film prepared from a CB PR with the addition of UV-SCA (a) before UV irradiation, (b) after UV irradiation, and (c) after post-baking. 
strength between the glass substrate and black film were schematically shown in Scheme 3. The criterion about the function of UV-SCA is to examine the decay ratio of adhesion strength before and after ITO sputtering process according to the following equation:

Decay ratio of adhesion strength (\%)

$$
=\frac{(\text { adhesion strength })_{\text {before ITo }}-(\text { adhesion strength })_{\text {after ITO }}}{(\text { adhesion strength })_{\text {before ITo }}} \times 100
$$

\section{Results and discussion}

\subsection{Synthesis of UV-curable silane-coupling agent}

In this work, UV-SCA was prepared by the urethane linkage reaction between $\mathrm{OH}$ groups of EPA and isocyanate group of ICPTES at $75^{\circ} \mathrm{C}$. The molar ratio of EPA to ICPTES was $1: 2$ and its structure was confirmed by FTIR as well as ${ }^{1} \mathrm{H}$ NMR analysis.

Fig. 1 shows the FTIR spectra of EPA and UV-SCA. It can be seen in the spectrum of UV-SCA that the absorbance of hydroxyl group between 3200 and $3600 \mathrm{~cm}^{-1}$ largely decreases compared to EPA due to the reaction with isocyanate of ICPTES. This reaction produces urethane group which exhibits two peaks at 3370 and $1540 \mathrm{~cm}^{-1}$. In addition, the alkoxy group of silane presenting a peak at $1080 \mathrm{~cm}^{-1}$ is also observed.

Figs. 2 and 3 show the ${ }^{1} \mathrm{H}$ NMR spectra of EPA and UV-SCA, respectively. All the characteristic peaks as well as peak areas of these two compounds are listed in Tables 1 and 2, respectively. The EPA structure was confirmed by ${ }^{1} \mathrm{H}$ NMR and the average $n$ value was calculated to be about 0.3. The detail in calculation of $n$ was illustrated in Table 1. Compared with EPA, the peak of hydroxyl group at $2.3 \mathrm{ppm}$ in the spectrum of UV-SCA largely decreases due to the reaction with isocyanate of ICPTES. This reaction produces $\mathrm{NH}$ group, which exhibits broad peaks between 4.8 and $5.0 \mathrm{ppm}$. In addition, the peaks of three protons of acrylate group at 5.9, 6.2, and $6.5 \mathrm{ppm}$ are also observed. According to the FTIR and ${ }^{1} \mathrm{H}$ NMR results, we believe that the UV-SCA was successfully prepared.
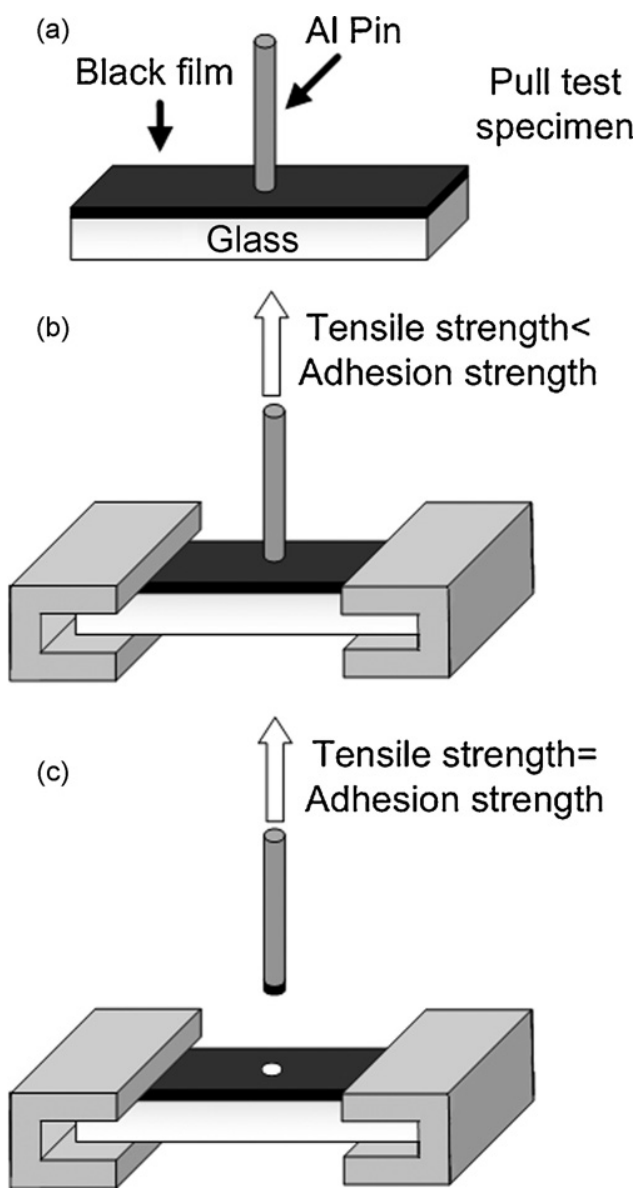

Scheme 3. (a) The pull test specimen. The test scheme to determine the adhesion strength between the glass substrate and black film in the states of (b) tensile strength < adhesion strength, and (c) tensile strength = adhesion strength.

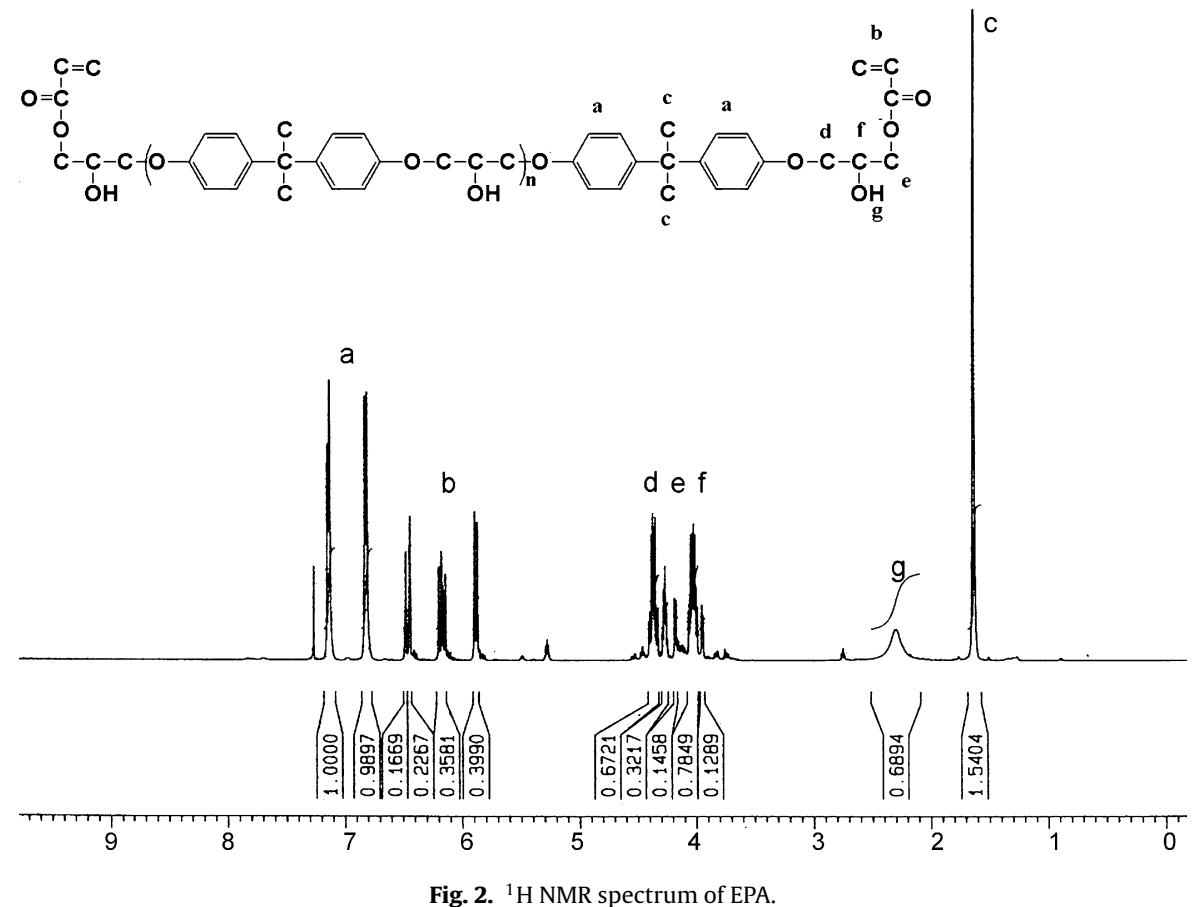

Fig. 2. ${ }^{1} \mathrm{H}$ NMR spectrum of EPA. 


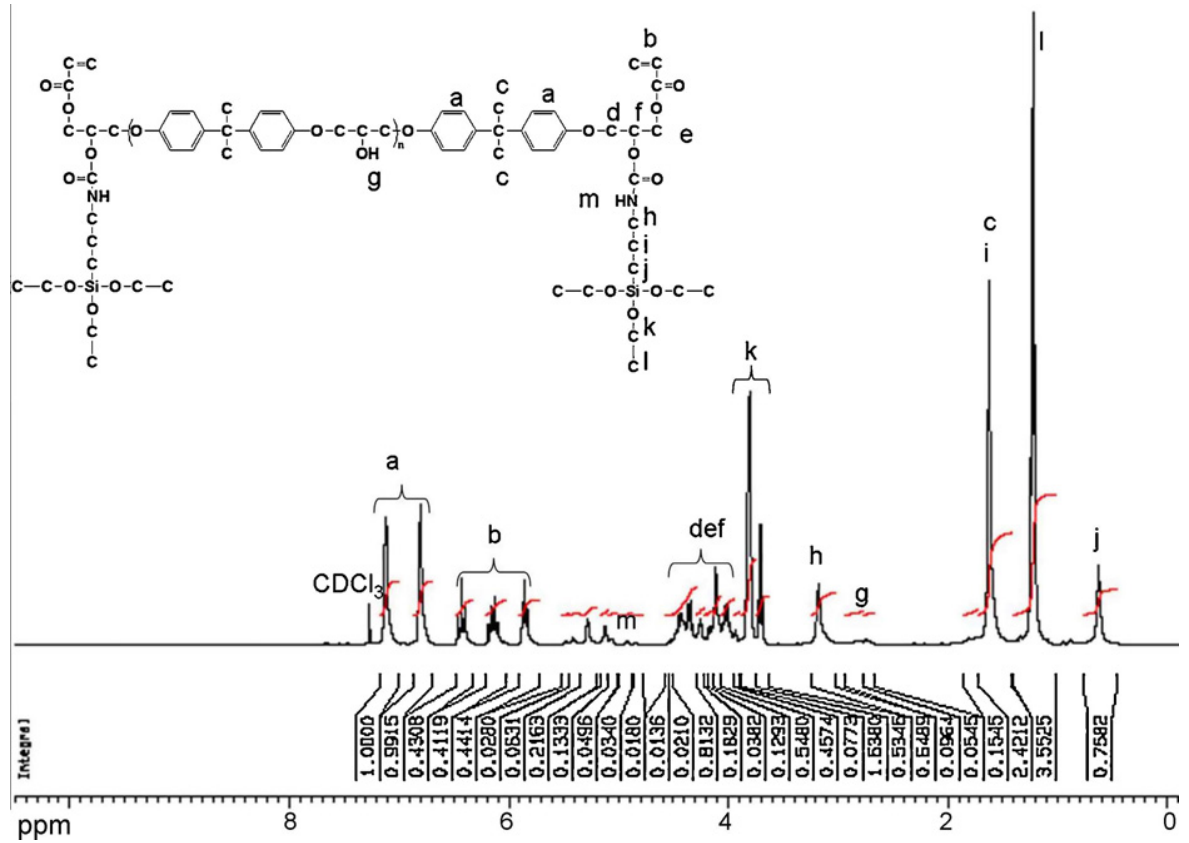

Fig. 3. ${ }^{1} \mathrm{H}$ NMR spectrum of UV-SCA.

Table 1

Chemical shift, number of hydrogen, and the calculation of $n$ in EPA

\begin{tabular}{|c|c|c|c|c|c|c|c|}
\hline Chemical shift & $6.8,7.2$ & $5.9,6.2,6.5$ & 1.7 & 4.4 & 4.2 & 4 & 2.3 \\
\hline Peak label & a & $\mathrm{b}$ & c & d & $\mathrm{e}$ & $\mathrm{f}$ & $\mathrm{g}$ \\
\hline No. of hydrogen & $8(1+n)$ & 6 & $6(1+n)$ & $5(2+n)$ & & & $2+n$ \\
\hline Peak area & 1.99 & 1.15 & 1.54 & 2.08 & & & - \\
\hline To calculate the $n$ in EPA & $6 /[8(1+$ & $n=0.3$ & & & & & \\
\hline
\end{tabular}

\subsection{Adhesion strength}

In this study, the UV-SCA was used as an adhesion promoter in order to improve the adhesion strength between black matrix and glass substrate after ITO sputtering process. The UV-SCA in black matrix was maintained at $1 \mathrm{wt} \%$, and the influence of UV-SCA on the adhesion strength between black matrix and glass substrate before and after ITO sputtering processes was discussed.

As shown in Fig. 4, before the ITO sputtering process, the adhesion strengths of samples are all higher than $400 \mathrm{~kg} \mathrm{~cm}^{-2}$; however, they are decreased after ITO sputtering process, especially in the sample without the addition of UV-SCA. Taking the CB PR of BK 5017 without the addition of UV-SCA for an example, the adhesion strength is decreased from $424 \mathrm{~kg} \mathrm{~cm}^{-2}$ before ITO sputtering process to $264 \mathrm{~kg} \mathrm{~cm}^{-2}$ after ITO sputtering process. The decay ratio of adhesion strength (shown in Fig. 5) is relatively high, about 38\%, which also confirms that the large decrease in adhesion strength results from the ITO sputtering process.

On the contrary, in the sample of BK 5017 with the addition of $1 \mathrm{wt} \% \mathrm{UV}-\mathrm{SCA}$, the decay ratio of adhesion strength decreases to about $23 \%$. The same behavior is also observed in the sample of ADK L4 with the addition of $1 \mathrm{wt} \% \mathrm{UV}-\mathrm{SCA}$, in which the decay ratio of adhesion strength is relatively low, only about $15 \%$, indicating

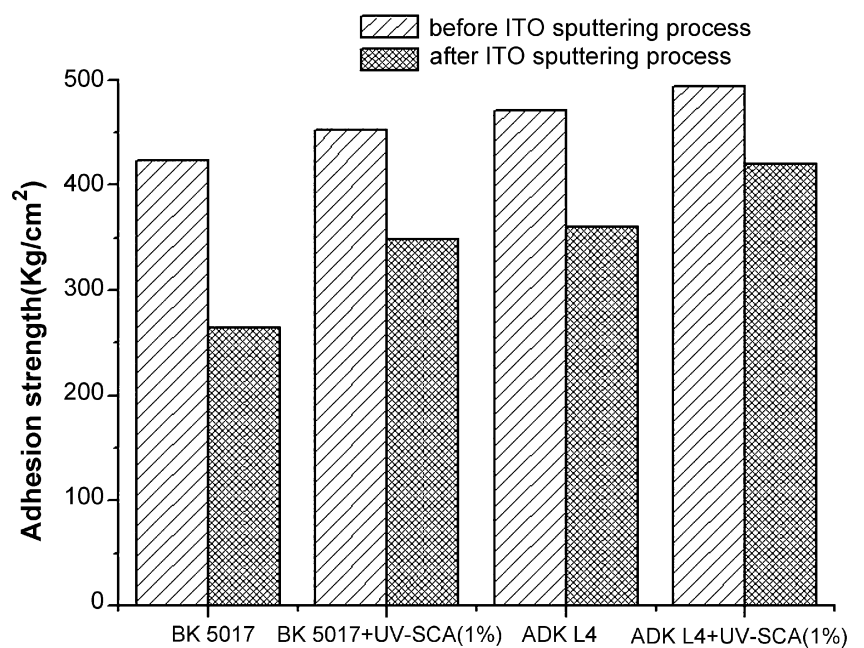

Sample codes

Fig. 4. The adhesion strengths of samples before and after ITO sputtering process.

Table 2

Chemical shift, number of hydrogen, and peak of area of UV-SCA

\begin{tabular}{|c|c|c|c|c|c|c|c|c|c|c|}
\hline Chemical shift & $6.8,7.1$ & $5.9,6.2,6.5$ & $1.4-1.7$ & $3.8-4.5$ & 2.8 & 3.2 & 0.5 & 3.7 & 1.2 & $4.8-5$ \\
\hline Peak label & a & $\mathrm{b}$ & $\mathrm{c}, \mathrm{i}$ & $d, e, f$ & $\mathrm{~g}$ & $\mathrm{~h}$ & $\mathrm{j}$ & $\mathrm{k}$ & 1 & $\mathrm{~m}$ \\
\hline No. of hydrogen & 10.4 & 6 & 11.8 & 11.5 & 0.3 & 4 & 4 & 12 & 18 & 2 \\
\hline Peak area & 1.99 & 1.28 & 2.42 & 2.28 & - & 0.65 & 0.76 & 2.17 & 3.55 & - \\
\hline
\end{tabular}




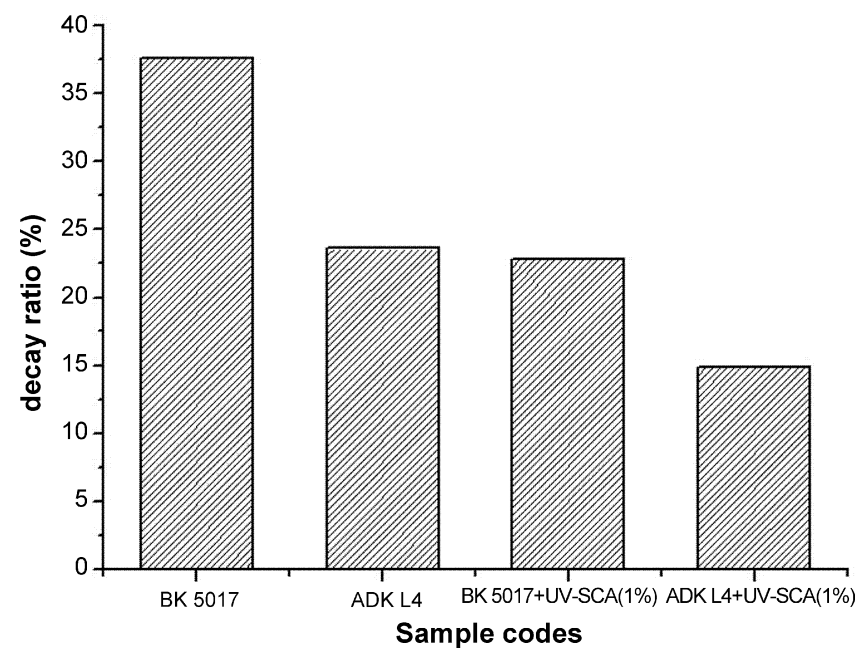

Fig. 5. The decay ratio of adhesion strength of samples after ITO sputtering process.

that the UV-SCA is an effective adhesion promoter. This is because the acrylate double bond of UV-SCA can copolymerize with the UVcurable and alkali-soluble resins to form covalent bonds in the black matrix; on the other hand, the alkoxy group of UV-SCA can react with glass substrate to produce a strong $\mathrm{Si}-\mathrm{O}-\mathrm{Si}$ bond. Therefore, the adhesion strength after ITO sputtering process was significantly improved.

\section{Conclusion}

A new UV-SCA was successfully prepared by the urethane linkage reaction between EPA and ICPTES. This UV-SCA can be used as an adhesion promoter in the black matrix application. From the experimental results, the adhesion strength between black matrix and glass substrate after ITO sputtering process was largely improved when $1 \mathrm{wt} \%$ UV-SCA was added in CB PR (based on the total solid of CB PR). This is because the acrylate double bond of UV-SCA can copolymerize with the UV-curable and alkali-soluble resins to form covalent bonds in the black matrix; on the other hand, the alkoxy group of UV-SCA can react with glass substrate to produce a strong $\mathrm{Si}-\mathrm{O}-\mathrm{Si}$ bond.

\section{Acknowledgement}

The authors gratefully acknowledge Allied Material Technology Corp. (Taiwan) for research fellowship in technology.

\section{References}

[1] E.P. Plueddmann, Silanes and other coupling agents, The Netherlands, VSP, Utrect: 1992; p 3-19.

[2] T.H. Chiang, T.E. Hsieh, J. Adhes. Sci. Technol. 19 (1) (2005) 1-18.

[3] G. Xu, M. Gong, W. Shi, Polym. Adv. Technol. 16 (6) (2005) 473-479.

[4] D. Bikiaris, P. Matzinos, J. Prinos, V. Flaris, A. Larena, C. Panayiotou, J. Appl. Polym. Sci. 80 (14) (2001) 2877-2888.

[5] D. Bikiaris, P. Matzinos, A. Larena, V. Flaris, C. Panayiotou, J. Appl. Polym. Sci. 81 (3) (2001) 701-709.

[6] M. Mohseni, M. Mirabedini, M. Hashemi, G.E. Thompson, Prog. Org. Coat. 57 (4) (2006) 307-313.

[7] W. Fu, H. Yang, S. Liu, M. Li, G. Zou, Mater. Chem. Phys. 100 (2-3)(2006) 246-250.

[8] K.D. Weaver, Polym. Compos. 16 (2) (1995) 161.

[9] J.R. Olson, D.E. Day, J.O. Stoffer, J. Compos. Mater. 26 (8) (1992) 1181.

[10] M.W. Ellsworth, B.M. Novak, Chem. Mater. 5 (6) (1993) 839-844.

[11] B.K. Coltrain, C.J.T. Landry, J.M. O'Reilly, A.M. Chamberlain, G.A. Rakes, J.S. Sedita, et al., Chem. Mater. 5 (10) (1993) 1445-1455.

[12] S. Lu, M.M. Melo, J. Zhao, E.M. Pearce, T.K. Kwei, Macromolecules 28 (14) (1995) 4908-4913.

[13] R. Joseph, S. Zhang, W.T. Ford, Macromolecules 29 (4) (1996) 1305-1312.

[14] T. Saitoh, A. Sekino, M. Hiraide, Chem. Lett. 33 (7) (2004) 912-913.

[15] H.B. Sunkara, J.M. Jethmalani, W.T. Ford, Chem. Mater. 6 (4) (1994) 362-364.

[16] E.C. Hagberg, M.W. Hart, L. Cong, C.W. Allen, K.R. Carter, J. Inorg. Organomet. P. 17 (2) (2007) 377-385. 